\title{
A decade of influence in the Singapore youth mental health landscape: the Community Health Assessment Team (CHAT)
}

Sekar S $\underline{\text { Harish }}{ }^{1}$, MBBS, Ganesh K $\underline{\text { Kundadak }^{1}}$, MBBS, MRCPsych, Yi Ping $\underline{\text { Lee }}^{1}$, Charmaine $\underline{\text { Tang }^{1}}$, MBBS, MRCPsych, Swapna K $\underline{\text { Verma }}^{1}$, MBBS, MD

\section{INTRODUCTION}

Adolescence and young adulthood are thought to be particularly tumultuous times in neuropsychological and physical development. Demographic studies show that this phase of life is associated with the emergence of mental disorders. ${ }^{(1)}$ The second Singapore Mental Health Study, conducted in 2016, revealed a significant association between younger age and mental disorders. In particular, young adults aged 18-34 years had the highest lifetime prevalence as well as 12 -month prevalence for any mental disorder. ${ }^{(2)}$ In 2017, it was reported that mental illness was the largest contributor to years lost to disease among young people aged 10-34 years in Singapore, and the second largest overall contributor across all age groups. ${ }^{(3)}$

While many psychiatric institutions offer services for children and adults, those in the young adult population may not find a catered service that is tailored to their specific needs. Increasing attempts are being made worldwide to identify the barriers to accessing mental health services that youths face, and to introduce dedicated mental health services for youths and young adults. ${ }^{(4)}$ There has also been an impetus to have a dedicated youth mental health service in Singapore. With support and funding from the Ministry of Health $(\mathrm{MOH})$, the Community Health Assessment Team (CHAT) was started in 2009. CHAT's assessment centre is located in a youth-friendly and easily accessible location in the heart of Singapore's retail district. Through its programmes targeting young persons aged $16-30$ years, CHAT aims to increase Awareness of mental health issues, Access to mental health resources, and to provide confidential and personalised mental health Assessments, as captured in its AAA model.

Currently in its tenth year of operation, CHAT has established itself as a leader in the youth mental health landscape in Singapore. This paper describes the progress and maturation of CHAT's strategic framework over the last decade, highlighting some of the key programmes that have been developed to provide a more comprehensive service to Singaporean youths, and presents clinical and sociodemographic data reflecting the ten-year trends in referrals and assessments.

\section{METHODS}

A 2014 study by Poon et al discussed the various objectives, action areas and strategies employed by CHAT. ${ }^{(5)}$ Table I provides an updated summary and new strategies that were developed within the last five years. Here, we describe in detail three key programmes, namely webCHAT, CHAT Supportive Interventions (CSIs) and CHAT Ambassadors Programme. CHAT maintains an administrative database where it tracks outcome indicators. The breakdown of these indicators, over the past ten years of CHAT's operation (April 2009 to March 2019), are presented and discussed in this paper.

\section{RESULTS}

Young persons who were referred to CHAT from April 2009 to March 2019 were included in this analysis. As shown in Fig. 1, a total of 6,173 individuals were referred to CHAT for assessment, of whom 3,343 (54.2\%) underwent a complete mental health assessment. The main reasons behind the non-assessment of 2,830 $(45.8 \%)$ individuals were that they were uncontactable, declined an in-person assessment, did not meet CHAT's age criteria or were already being seen by a psychiatrist while awaiting CHAT assessment. These individuals were sent a text message to return the call, advised to re-refer themselves when they were ready for an assessment, directed to more appropriate mental health services or advised to consult their respective psychiatrists.

Fig. 2 shows the source of the CHAT referrals. Self-referrals made up the vast majority, accounting for $73.9 \%$ of all referrals. The median age of the referred persons was 19 years. $70.1 \%$ of all young persons referred to CHAT were female; females made up $75.6 \%$ of all self-referrals to CHAT and $68.6 \%$ of all those who completed their assessments. However, referrals from other sources were more evenly distributed with only $54.7 \%$ being female.

Fig. 3 shows the breakdown of the assessment impressions of those who underwent a formal mental health assessment with CHAT. $23.2 \%$ of young persons assessed were ascertained to have no mental illness and 2,568 (76.8\%) young persons were assessed as having mental health issues, with mood disorder being the most commonly rendered diagnosis $(36.1 \%)$.

At the end of the assessment, those deemed to require further interventions were provided with downstream referrals to a spectrum of services. $5.5 \%$ of young persons were assessed as not requiring a follow-up, while $3.9 \%$ declined the recommendations offered to them. The remaining $90.6 \%$ of those assessed, including 
Table I. CHAT's strategic framework.

\begin{tabular}{|c|c|c|}
\hline Objectives & Action areas & Strategies \\
\hline $\begin{array}{l}\text { - Increase accessibility to mental health } \\
\text { services } \\
\text { - Streamline referral process for young } \\
\text { persons to access mental health } \\
\text { services }\end{array}$ & $\begin{array}{l}\text { Access to specialist } \\
\text { support }\end{array}$ & $\begin{array}{l}\text { - Free and confidential mental health assessment service offered by mental } \\
\text { - healthcare professionals in a central location } \\
\text { - Referral to and coordination with appropriate downstream community } \\
\text { services for young persons who have undergone assessments } \\
\text { - Referral for subsidised care in public hospitals } \\
\text { - CHAT Supportive Interventions to bridge the gap between assessment and } \\
\text { specialist support* } \\
\text { - Enabling greater access to CHAT through webCHAT, an online mental health } \\
\text { service* }^{*}\end{array}$ \\
\hline \multirow[t]{3}{*}{$\begin{array}{l}\text { - Raise awareness regarding youth } \\
\text { mental health concerns } \\
\text { - Encourage use of mental health } \\
\text { services and resources in young } \\
\text { persons }\end{array}$} & Public health target & $\begin{array}{l}\text {-Wider outreach programmes, community public awareness projects, school } \\
\text { and community initiatives } \\
\text { - An online presence via our website (https://www.chat.mentalhealth.sg) and } \\
\text { social media, including Facebook (http://www.facebook.com/chatfans) and } \\
\text { Instagram (@chatfans) } \\
\text { - Media coverage of services and projects } \\
\text { - Working with ministries and governmental organisations to co-create youth } \\
\text { mental health services* }\end{array}$ \\
\hline & Capability building & $\begin{array}{l}\text {-Training community partners to identify signs of distress, support young } \\
\text { persons to seek help and provide resources on avenues of help } \\
\text { - Networking with and supporting community partners and academic/ } \\
\text { vocational institutions in the management of young persons with mental } \\
\text { health issues }\end{array}$ \\
\hline & $\begin{array}{l}\text { Youth participation } \\
\text { and outreach }\end{array}$ & $\begin{array}{l}\text { - Supporting initiatives from young persons, for example, in school projects } \\
\text { and roadshows } \\
\text { - Enabling and empowering young persons in youth mental health advocacy } \\
\text { through the CHAT Ambassadors Programme* } \\
\text { - Engaging young persons in focus group discussions* } \\
\text { - Involving young persons in CHAT's service development* }\end{array}$ \\
\hline
\end{tabular}

*New strategies that were developed within the last five years. CHAT: Community Health Assessment Team

some who were ascertained as not having a mental illness, were referred to the most appropriate and relevant organisations based on their individual needs. $24.9 \%$ of young persons were referred to social service organisations (SSOs) for community counselling. $33.5 \%$ of young persons were referred to outpatient psychiatric services, with $20.0 \%$ of referrals being made to restructured hospitals and $13.5 \%$ to the Institute of Mental Health. $12.0 \%$ of young persons were referred to school counsellors and a further $5.1 \%$ of young persons were referred for brief supportive interventions at CHAT.

webCHAT is an online screening service run by CHAT that was started in 2017. Its objective is to make mental health support accessible to young people who may be apprehensive of telephone or in-person consultations. Through webCHAT, young persons can have a live online conversation with a mental health professional. Clients can choose to be anonymous and can join and leave the chat portal at any time.

A total of 459 webCHAT sessions were conducted from June 2017 to March 2019. The anonymous nature of this modality makes it difficult to track if the same individual has had multiple sessions. 54 (11.8\%) sessions involved general enquiries on CHAT or those on broader mental health services available in Singapore. Out of the 459 webCHAT sessions, 354 $(77.1 \%)$ individuals stayed online in the session long enough for a preliminary assessment to be conducted. These individuals were provided recommendations for subsequent follow-ups: $193(54.5 \%)$ individuals did not require a follow-up or declined the recommendation; $82(23.2 \%)$ were determined to require a CHAT intervention, either via a phone call or an in-person consultation; 50 (14.1\%) were recommended to follow up at family service centres or voluntary welfare organisations; and 29 $(8.2 \%)$ were referred to specialists or other services. The severity of mental health distress and functioning experienced by users of the webCHAT service is typically assessed using the Global Assessment of Functioning (GAF) scale, and a GAF-based mental health screening was completed for 241 young persons. Of these, $211(87.6 \%)$ yielded a positive result for mental health issues.

One of CHAT's primary goals is to increase timely access to specialist mental health support for youths in distress. Despite the success of CHAT in providing avenues to help young people in distress, barriers still exist in their journey to recovery, including a long wait time for first appointments with tertiary care and limited dedicated youth mental health services that can support young people who seek only counselling. To address this gap, CSI is an on-site counselling service delivered by certified solution-focused therapy practitioners to provide brief (up to six sessions) support for distressed individuals who are facing a long wait for their first appointment at specialist psychiatric services, and are either not agreeable to or not suitable for specialist services. The Outcome Rating Scale, designed and normed for adults and adolescents (age 


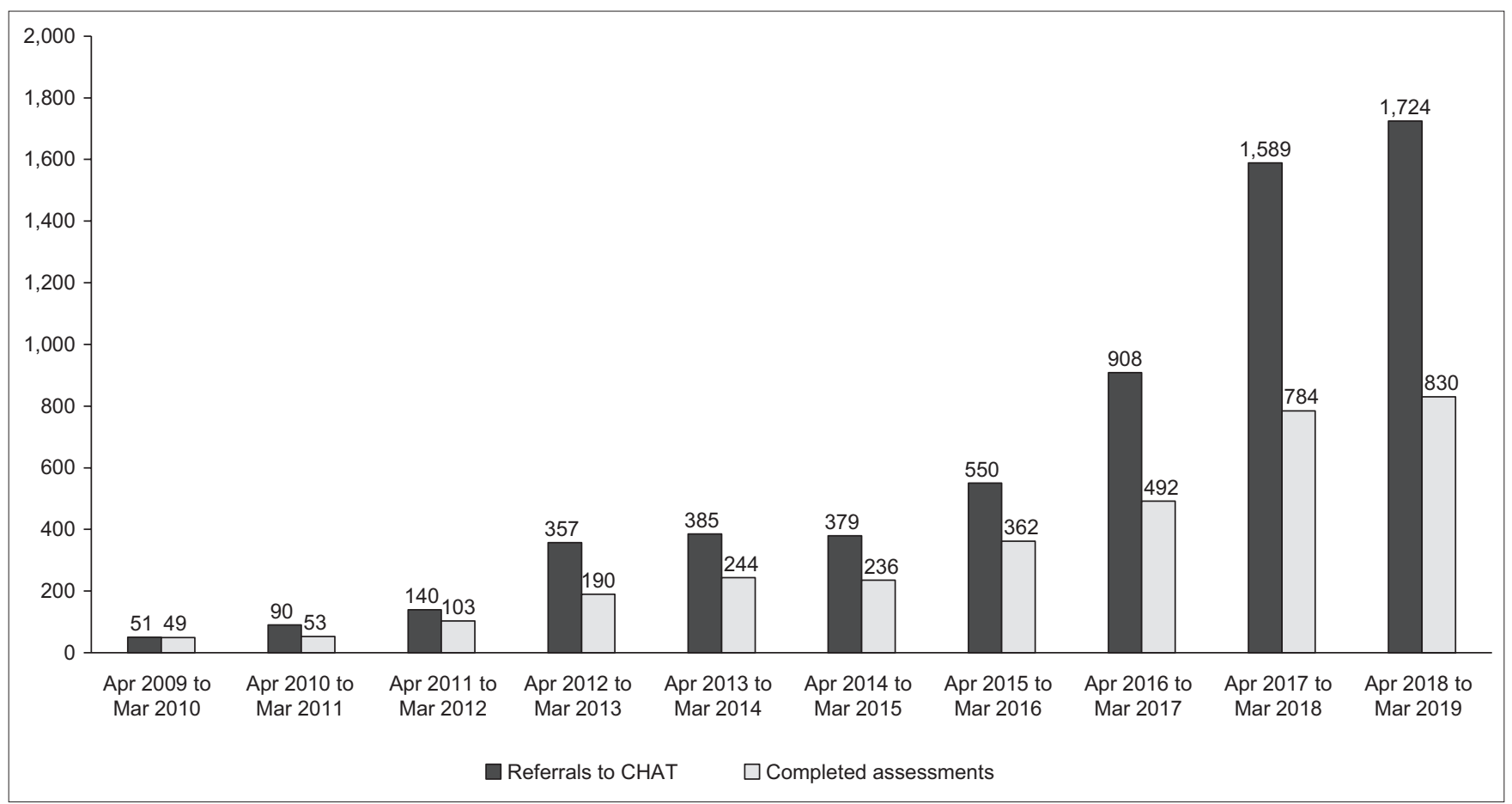

Fig. 1 Graph shows the annual number of CHAT referrals $(n=6,173)$ and assessments $(n=3,343)$ between April 2009 and March 2019. CHAT: Community Health Assessment Team

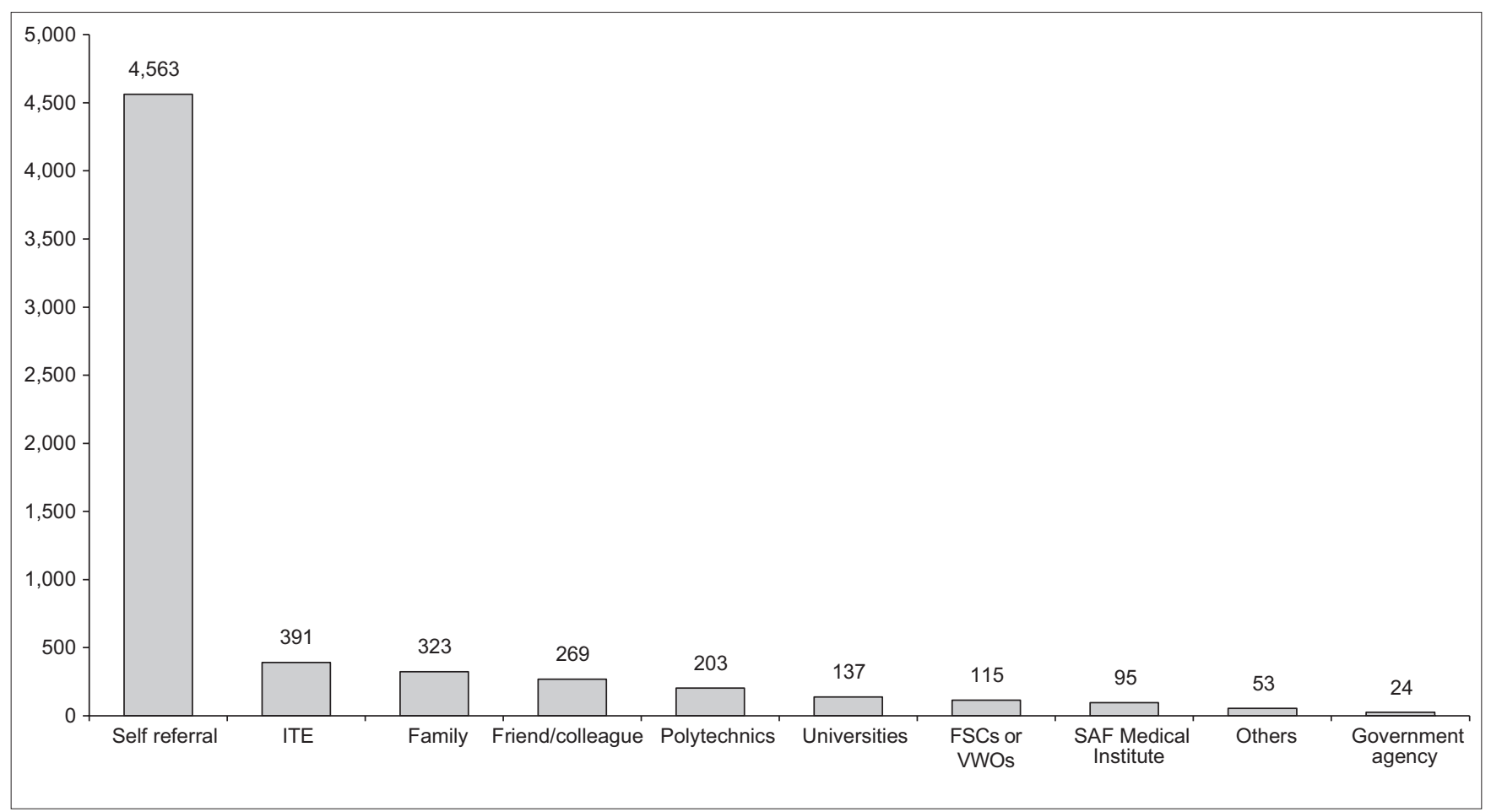

Fig. 2 Graph shows the sources of referrals to CHAT between April 2009 and March 2019 ( $n=6,173$ ). CHAT: Community Health Assessment Team; FSC: family service centre; ITE: Institute of Technical Education; SAF: Singapore Armed Forces; VWOs: volunteer welfare organisations

$\geq 13$ years), was used to track the well-being of young people on a per-session basis. Since July 2015, 115 young people assessed through CHAT have benefitted from CSI. Of these, 54 (47.0\%) reported that their distress was reduced by more than $25 \%$, while $23(20 \%)$ experienced a 6\%-25\% reduction in distress. 38 (33\%) young people whose distress was reduced by $5 \%$ or less were supported by CHAT with referrals to relevant services. Out of 57 young people who responded to a satisfaction survey regarding their experience with CSI, 53 (93.0\%) stated 'almost all or most of my needs have been met', and 53 (93.0\%) felt 'very or mostly satisfied' with CSI. All respondents stated that they would 'definitely or generally' recommend CHAT to their distressed friends.

The CHAT Ambassadors Programme was started in 2014 in order to augment outreach efforts and increase youth participation. 


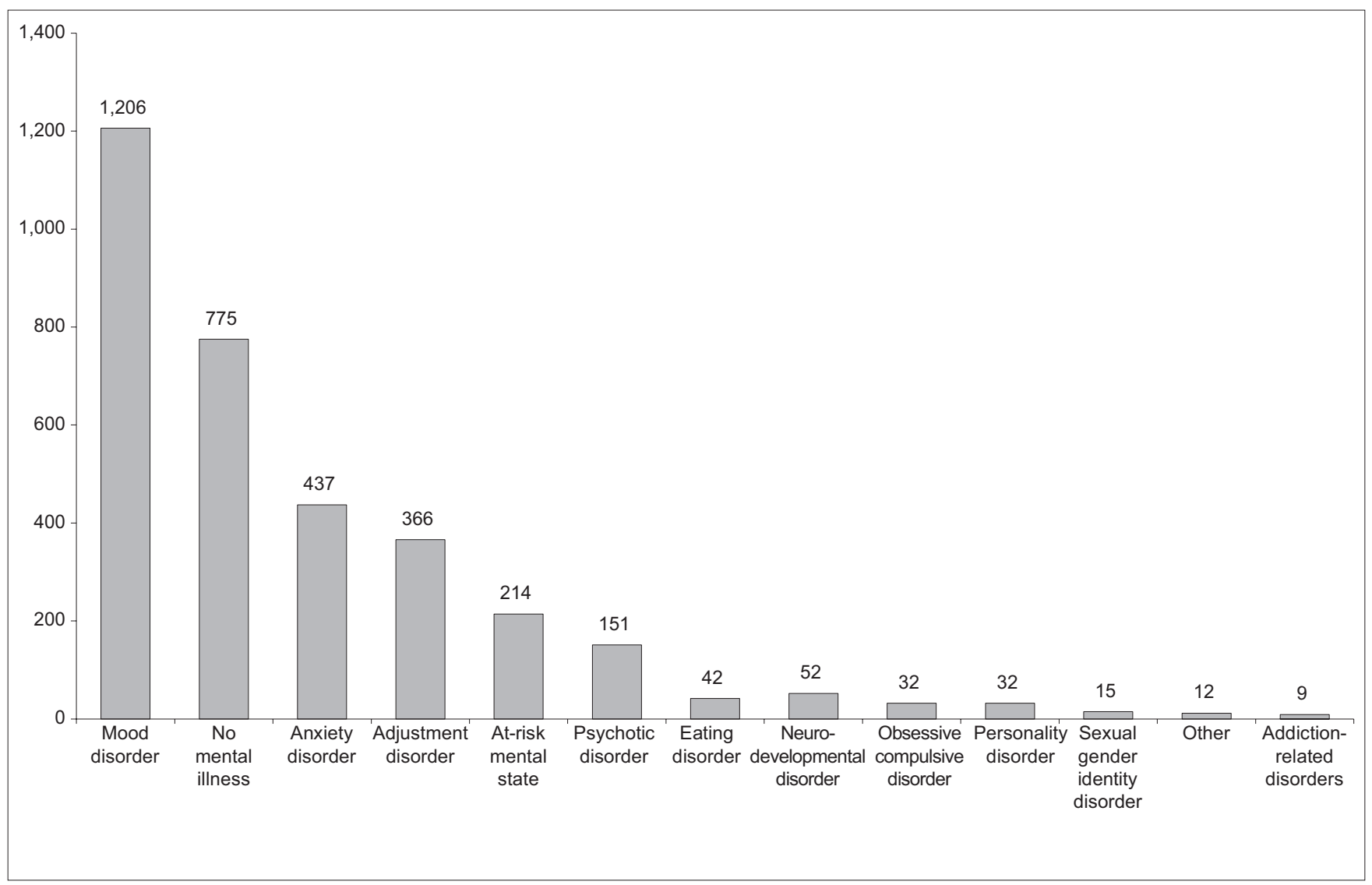

Fig. 3 Graph shows the breakdown of CHAT assessment impressions between April 2009 and March 2019 ( $n=3,343$ ). CHAT: Community Health Assessment Team

The programme is a volunteer-based youth participatory group for individuals aged between 16 years and 30 years. The ambassadors are young people who have demonstrated an openness towards collaborating with fellow youths and are passionate about improvement of youth mental health service and advocacy work. The ambassadors are empowered to initiate their own service improvement projects that are aligned with $\mathrm{CHAT}^{\prime}$ s strategic goals. They are encouraged to take charge of the implementation of the projects initiated by CHAT staff members. They also act as advocates for young people, providing a young person's perspective on issues, subsequently guiding CHAT's public outreach efforts and training programmes. Structured and on-thejob training is provided to the ambassadors in the areas of project management, outreach, peer helping and crisis management. From December 2014 to date, 55 CHAT ambassadors have been recruited and have successfully proposed and implemented eight service improvement initiatives, including the revamp of CHAT website and Hub space; refinement of CHAT's assessment service feedback form; development of a session summary and care package that includes post-assessment of psychoeducational materials; and development of various social media campaigns, experiential exhibitions and workshops.

\section{DISCUSSION}

The aforementioned results are a testament to the success of CHAT's strategies as a youth mental health service in Singapore. The referrals to CHAT, assessments rendered and subsequent downstream referrals made have increased exponentially over the past decade. Key initiatives including webCHAT, CSI and the CHAT Ambassadors Programme have certainly played a major role in $\mathrm{CHAT}^{\prime}$ s success. Other reasons include the ease of access to $\mathrm{CHAT}^{\prime}$ 's services, privacy and autonomy given to young people seeking help, as well as CHAT's partnerships with tertiary psychiatric services and community mental health resources. In a previous study that evaluated the extent to which $\mathrm{CHAT}^{\prime}$ s service model is youth-friendly using the World Health Organization youth-friendly heath service framework of accessibility, acceptability and appropriateness, the authors conducted a 27item survey involving 300 young people who had completed mental health assessments with CHAT, and found very high satisfaction rates, with $98.7 \%$ of respondents indicating that they were 'somewhat' or 'very satisfied' with the help offered at the end of the assessment. More than $90 \%$ of the respondents indicated that the service was accessible, acceptable and appropriate. This was achieved through the ease of commuting to the physical location, an easy referral process, affordability (accessibility), having a safe environment for assessment, offering privacy and confidentiality, staff being professional yet friendly and approachable, staff offering information and giving choices to young people (acceptability), providing recommendations regarding treatment and help options fitting with the young person's concerns, and making timely referrals to these options (appropriateness). ${ }^{(6)}$

In 2014, a service review paper by Poon et al described the beginnings of $\mathrm{CHAT}^{(5)}$ In the subsequent years, there has been 
greater public awareness regarding CHAT and other mental health services. This is evidenced by the increase in the proportion of self-referrals to CHAT from 40.1\% between 2009 and 2013 to $73.9 \%$ at present. Along with the increase in self-referrals, we have also noted the presence of a gender gap among those referred to CHAT. While referrals made by counsellors, educational institutions and healthcare institutions have been more even in their gender distribution ( $54.7 \%$ vs. $45.3 \%$ ), there is a much wider disparity in the self-referrals to CHAT (75.6\% vs. $24.4 \%$ ). Females are approximately three times more likely than males to seek help at CHAT. In contrast, local epidemiological studies have shown that males in Singapore were more likely than females to experience a mental disorder in their lifetime. ${ }^{(2)}$ Other studies have also found gender differences in health-seeking behaviours among youth. ${ }^{(7)}$ This gap is an action area that the CHAT team has identified and attempted to address in their current outreach programmes as well as in future efforts.

There have also been shifts in the diagnoses rendered at the first assessment. While psychotic disorders and prodromal states were the most commonly rendered diagnoses (27.6\%) from 2009 to 2013, mood disorders are the most commonly rendered diagnosis at present $(36.1 \%){ }^{(5)}$ This could be attributable to the fact that, similar to the Australian model, CHAT was initially established as a continuum of the Singapore Early Psychosis Intervention Programme (EPIP), with its original focus being to address the stigma related to schizophrenia and to reduce the duration of untreated psychosis. Increasing awareness regarding EPIP and the similar target age group would lead to many young persons being directly seen by the EPIP service rather than being referred to CHAT first. The current diagnosis distribution is more reflective of the mental health concerns faced by today's youths.

The results also showcase an increase in the variety of downstream referrals from CHAT as compared to the first four years of its operation. From 2009 to 2013, 61.5\% of young persons were referred to speciality outpatient clinics and hospitals, while another $28.6 \%$ were referred to community or school counselling services. By 2019, these proportions had decreased significantly, and the services that young persons were referred to became more diverse. CHAT has been working together with more mental health partners who might be better able to serve the needs of its users. This was in line with the national mental health blueprint, as laid out by $\mathrm{MOH}$, which emphasised the shifting of psychiatric care into the community. ${ }^{(8)}$

CHAT remains committed to improving its services and better connecting with youths in Singapore. As CHAT continues to grow as a youth mental health service, it endeavours to expand its current capabilities as well as to venture into new areas. There are plans to expand webCHAT to provide e-counselling for distressed young persons as an intermediary measure before they can access in-person services. For those who might be unwilling to speak to a member of the CHAT staff in person, on the phone or online through webCHAT, CHAT plans to introduce self-assessment and e-training resources on its website. CHAT also plans to go beyond being a primary provider of youth mental health services to training other organisations to provide youth mental health support. This includes other SSOs as well as businesses and government agencies that engage directly with young persons, as clients or employees.

As evidenced by this paper, CHAT is instrumental not only in providing youth mental health services, but also in uncovering the various barriers that exist in providing it. By focusing on the most established youth mental health service in Asia, this paper may serve as guidance for other Asian nations keen on embarking upon this field.

\section{REFERENCES}

1. Kessler RC, Berglund P, Demler O, et al. Lifetime prevalence and age-ofonset distributions of DSM-IV disorders in the National Comorbidity Survey Replication. Arch Gen Psychiatry 2005; 62:593-602.

2. Subramaniam M, Abdin E, Vaingankar JA, et al. Tracking the mental health of a nation: prevalence and correlates of mental disorders in the second Singapore mental health study. Epidemiol Psychiatr Sci 2019; 29:e29.

3. Epidemiology \& Disease Control Division, Ministry of Health, Singapore; Institute for Health Metrics and Evaluation. The Burden of Disease in Singapore, 1990-2017: An overview of the Global Burden of Disease Study 2017 results. Seattle, WA: IHME, 2019.

4. Hetrick SE, Bailey AP, Smith KE, et al. Integrated (one-stop shop) youth health care: best available evidence and future directions. Med J Aust 2017; 207:S5-S18.

5. Poon LY, Tay E, Lee YP, Lee H, Verma S. Making in-roads across the youth mental health landscape in Singapore: The Community Health Assessment Team (CHAT). Early Interv Psychiatry 2016; 10:171-7.

6. Lee YP, Ngaiman NKB, Poon LY, et al. Evaluating Singapore's CHAT assessment service by the World Mental Health Organisation (WHO) "youth-friendly" health services framework. Front Psychiatry 2019; 10:422.

7. Mission Australia and Black Dog Institute. Youth mental health report: youth survey 2012-16. Available at: https://apo.org.au/sites/default/files/resourcefiles/2017-04/apo-nid75809.pdf. Accessed April 22, 2021.

8. Institute of Mental Health, Singapore. National Mental Health Blueprint. Available at: https://www.imh.com.sg/page.aspx?id=129. Accessed April 22, 2021. 